\title{
Représentativité des températures mesurées dans la station météorologique Paris-Montsouris
}

\author{
Salem Dahech ${ }^{1,2 *}$, Sami Charfi ${ }^{2}$ et Malika Madelin ${ }^{1}$ \\ ${ }^{1}$ Université de Paris, UMR 8586 PRODIG, F-75000 Paris, France \\ ${ }^{2}$ Université de Sfax, laboratoire SYFACTE, Sfax, Tunisie
}

\begin{abstract}
Résumé - Cette étude s'intéresse à la représentativité des températures mesurées par la station météorologique de Paris-Montsouris ; elle montre que le site a un effet remarquable. En effet, l'ombre créée par les arbres situés à proximité des capteurs induit une baisse de la température avoisinant $2^{\circ} \mathrm{C}$ en moyenne durant l'été, entre $13 \mathrm{~h}$ et $18 \mathrm{~h}$ TU, par rapport aux stations d'Orly et de Vélizy, situées en milieu dégagé. La rugosité en ville ralentit la vitesse du vent de $1,5 \mathrm{~m} / \mathrm{s}$ et engendre une baisse de l'humidité entre $16 \mathrm{~h}$ et $19 \mathrm{~h}$ TU. La campagne de mesure itinérante nocturne, réalisée la nuit du 29 au 30 juin 2019 entre 23 h et $1 \mathrm{~h}$ TU, par temps caniculaire, montre que le parc est en moyenne $4^{\circ} \mathrm{C}$ plus frais que le centre de Paris et que l'intensité de l'îlot de chaleur urbain (ICU) atteint environ $11^{\circ} \mathrm{C}$ vers $1 \mathrm{~h} \mathrm{TU}$ du matin. La partie topographiquement surélevée du parc Montsouris est plus chaude d'environ $2^{\circ} \mathrm{C}$ que celle vallonnée vers $00 \mathrm{~h}$ TU.
\end{abstract}

Mots-clés : station météorologique / Paris-Montsouris / température / effet de site / ombre.

\begin{abstract}
Representativeness of the temperatures measured in the Paris-Montsouris weather station. This study focuses on the representativeness of temperatures measured by the Paris-Montsouris weather station; it shows that the site has a remarkable effect. Indeed, the shade created by the trees located near the sensors brings about an average drop in temperature of around $2^{\circ} \mathrm{C}$ during the summer, between 1 p.m. and 6 p.m. UT, compared to the Orly and Vélizy stations, located in an open environment. Roughness in the city slows wind speed by 1.5 $\mathrm{m} / \mathrm{s}$ and induces a drop in humidity between 4 p.m. and 7 p.m UT. The nocturnal car survey which was carried out in the night of June 29th to 30th 2019, by hot weather, shows that the Park is cooler than the center of Paris with an average of about $4^{\circ} \mathrm{C}$. The car survey method equally shows that the intensity of the urban heat island (UHI) reaches approximately $11^{\circ} \mathrm{C}$ around $1 \mathrm{a}$ am. UT. At midnight, on a clear day, the topographically elevated part of Montsouris Park is warmer by around $2^{\circ} \mathrm{C}$ than the hilly part.
\end{abstract}

Keywords: weather station / Paris-Montsouris / temperature / site effect / shade.

* Auteur de correspondance $:$ salem.dahech@univ-paris-diderot.fr

\section{Introduction}

Plusieurs études en topoclimatologie et particulièrement en climatologie urbaine se sont intéressées à la variation spatiotemporelle de la température à méso-échelle et à l'échelle locale (Carrega, 1994 ; Lemonsu et al., 2013 ; Masson et al., 2013 ; Charfi et Dahech, 2018 ; Oliveros et al., 2019). L'îlot de chaleur urbain (ICU) est parmi les phénomènes les plus étudiés en climatologie urbaine de par ses conséquences sur la consommation énergétique et sur la santé, notamment durant les vagues de chaleur (Lowe, 2016 ; Li et al., 2019). Par ailleurs, d'autres travaux ont analysé la relation entre température et pollution de l'air, entre autres les pics d'ozone troposphérique durant la saison photochimique (Martin, 2008 ; Santamouris, 2014) ainsi que l'effet de la végétation 
comme solution pour réduire le surchauffement urbain (Huyang et al., 1987 ; Charfi et al., 2014).

L'ICU est défini comme l'écart thermique entre la ville, densément bâtie, et la campagne, plus végétalisée ; il se manifeste par temps calme, la nuit (Oke, 1982). L'intensité de l'ICU se calcule souvent en se référant aux données fournies par deux stations du réseau de mesures météorologiques conventionnelles : une urbaine et une rurale (Escourrou, 1991 ; Cantat, 2004). La fiabilité de ces données (températures) peut être affectée par plusieurs facteurs tels que l'étalonnage des capteurs, le type d'abri, l'enregistrement des données mais aussi le déplacement des instruments de mesure et le changement de leur environnement immédiat (Lockhart, 2003). De plus, les températures mesurées en un lieu donné dépendent, en partie, de l'environnement immédiat de la mesure. En effet, plusieurs études ont montré les effets de l'occupation du sol sur les températures (Huang et al., 1987; Taha, 1997; Golden, 2004 ; Weng et al., 2004). L'air froid s'accumule dans les zones vallonnées ou derrière un obstacle, comme le remblai d'un chemin de fer (Beltrando et al., 2008). Une homogénéisation des données issues de plusieurs stations voisines s'avère nécessaire avant de réaliser des traitements statistiques comme l'étude de la tendance (Gubler et al., 2017).

L'extension des surfaces anthropisées explique, en partie, la tendance à la hausse des températures observée depuis trois décennies. Par exemple, d'après Douguedroit et Bridier (2007), une partie du réchauffement observé en région méditerranéenne française est causée par l'évolution de l'environnement des stations de mesures. De même, Charfi (2012) et Dahech (2013) ont montré que la hausse des températures constatée durant les trois dernières décennies, dans les agglomérations de Tunis, Sfax et Kairouan, dépasse largement celle observée dans les stations rurales de la Tunisie.

Dans le présent travail, nous nous intéressons aux modifications de la température induites par l'environnement immédiat de la station météorologique de Paris-Montsouris. Nous mettons l'accent sur l'effet de l'ombre, de l'urbanisation et la forme du relief sur la température. Les paramètres précités peuvent jouer sur la température mesurée, surtout par temps radiatif, à différents moments de la journée. Pour mettre en avant leurs effets, nous comparons les données recueillies à ParisMontsouris à d'autres stations non influencées par les facteurs précités. En complément, nous avons réalisé des mesures fixes et itinérantes dans le parc et ses alentours et à l'échelle de l'agglomération parisienne.

Il nous a paru important de nous intéresser à la station de Paris-Montsouris car elle est considérée comme la mémoire du climat parisien. En effet, elle fait partie des trois stations Météo France centenaires reconnues et labélisées par l'OMM en 2017. Elle est considérée parmi les soixante stations au monde offrant de longues séries de données homogènes, souvent utilisées pour étudier la variabilité climatique en France et en Europe de l'Ouest (Girault et Kounkou-Arnaud, 2018).

\section{Données et méthodes}

Nous comparons les températures horaires enregistrées à Paris-Montsouris à celles relevées dans deux autres stations situées à moins de $15 \mathrm{~km}$ plus au sud : Orly et Vélizy, gérées toutes les trois par Météo France (figure 1A). Le milieu environnant des stations de mesures est étudié, dans ce travail, à partir d'images satellitaires récentes à très haute résolution disponibles sur les sites de Google Earth et Google Maps, vérifiées et complétées par des visites de terrain. Ces images permettent de quantifier le pourcentage du bâti et de la végétation à différentes distances des capteurs (figure 2). Les fiches de métadonnées des stations sont également examinées pour vérifier la présence de l'ombre portée en fonction de la hauteur du soleil ainsi que la distance aux bâtiments. Les informations, fournies par Météo France, sont valables pour la période 1998-2017, soit la chronique sélectionnée pour comparer les températures des trois stations.

La station de Paris-Montsouris est implantée sur une petite colline à une altitude de $75 \mathrm{~m}$. Les températures sont influencées par l'effet de l'ombre et par des bâtiments, ainsi que par une étendue d'eau, situés à une distance de 10 à $30 \mathrm{~m}$. À Orly, la station est située sur un sol plat à altitude comparable $(84 \mathrm{~m})$; le thermomètre est influencé par des bâtiments situés entre 30 et 100 m. À Vélizy, l'altitude augmente de $100 \mathrm{~m}$ par rapport aux deux 
autres stations (174 m) mais les caractéristiques du site sont semblables à celle d'Orly. Dans les trois stations, la hauteur de la pelouse est inférieure à 25 $\mathrm{cm}$ et l'incertitude globale des capteurs est de $0,15^{\circ} \mathrm{C}$ (source : métadonnées de Météo France). Des thermomètres à résistance de platine sont utilisés dans les trois stations, des sondes PT100 sont couramment utilisées. Dans un rayon de $1 \mathrm{~km}$ autour des stations de Vélizy, Orly et Montsouris, les pourcentages des surfaces bâties (sans compter les routes) sont respectivement de 4,5, 8 et $27 \%$ (figure 1). Il est important de noter également que le volume des bâtiments est largement plus important à proximité du parc Montsouris.
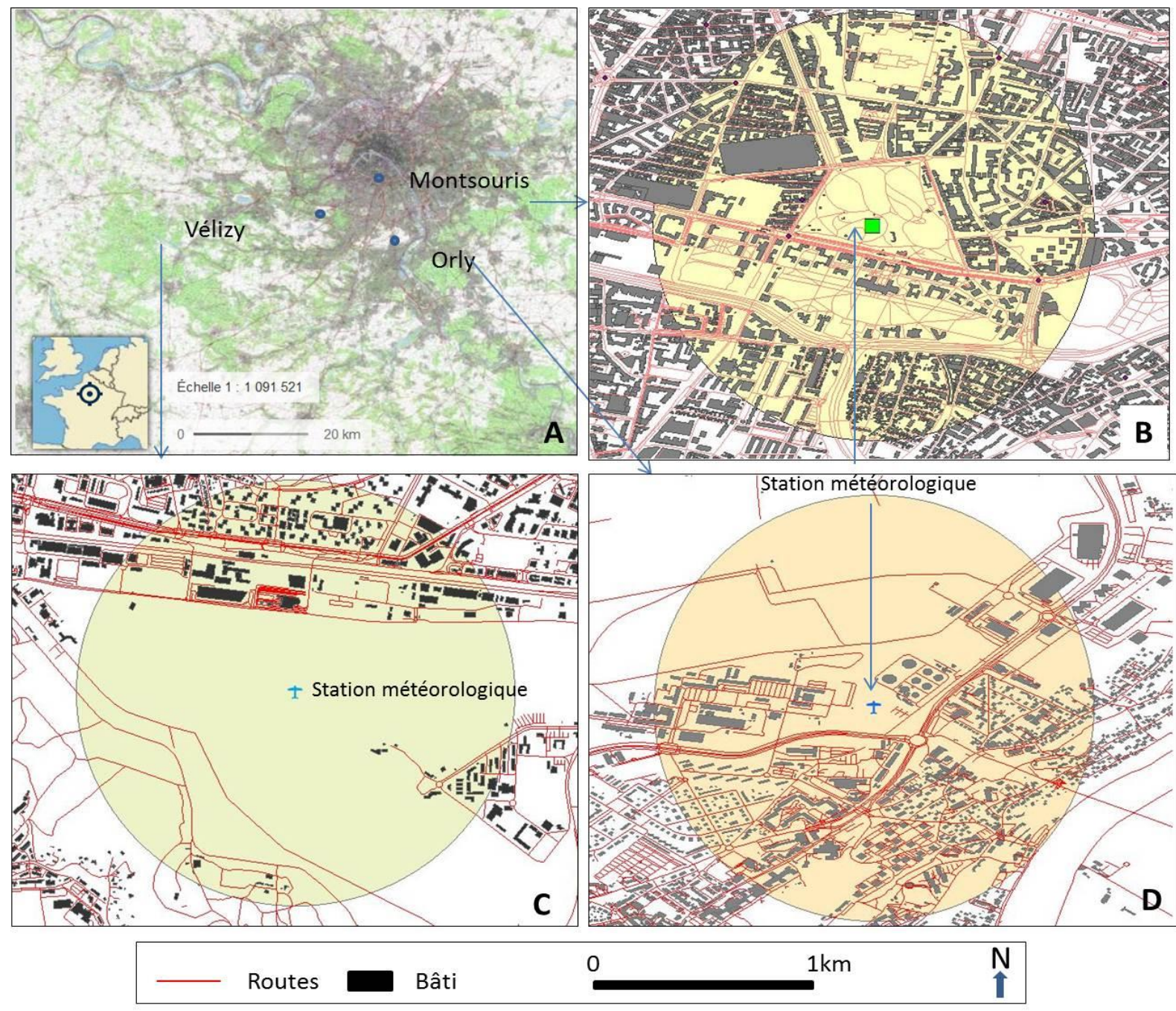

Figure 1. Localisation des stations météorologiques $(A)$ et répartition du bâti dans un rayon de $1 \mathrm{~km}$ autour des stations de Paris-Montsouris (B), Vélizy (C) et Orly (D) ; fond de la carte A importé de Géoportail ; bâti et routes importés de Google Maps pour 2019 sur B, C et D. Weather station location $(A)$ and building areas within a radius of one $\mathrm{km}$ around the stations of Paris Montsouris (B), Vélizy $(C)$ and Orly (D); background of map A imported from Géoportail; buildings and roads imported from Google Maps 2019 on B, C and D).

Le cycle journalier moyen des températures enregistrées en été (juin, juillet, août) par temps clair et vent faible (couverture nuageuse < à 2 octas et vent $<4 \mathrm{~m} / \mathrm{s}$ ) pendant la période 1998-2017, est calculé et confronté par la suite au cycle moyen des jours perturbés (vent fort $>8 \mathrm{~m} / \mathrm{s}$ et ciel couvert $>6$ octas). Nous étudions également le cycle journalier moyen des températures enregistrées en hiver, toutes situations météorologiques confondues. En complément, nous comparons le vent et l'humidité (exprimée par la température du point de rosée), enregistrés à Montsouris et aux deux autres stations pour regarder une éventuelle implication de ces deux paramètres météorologiques dans l'explication des écarts de températures. 


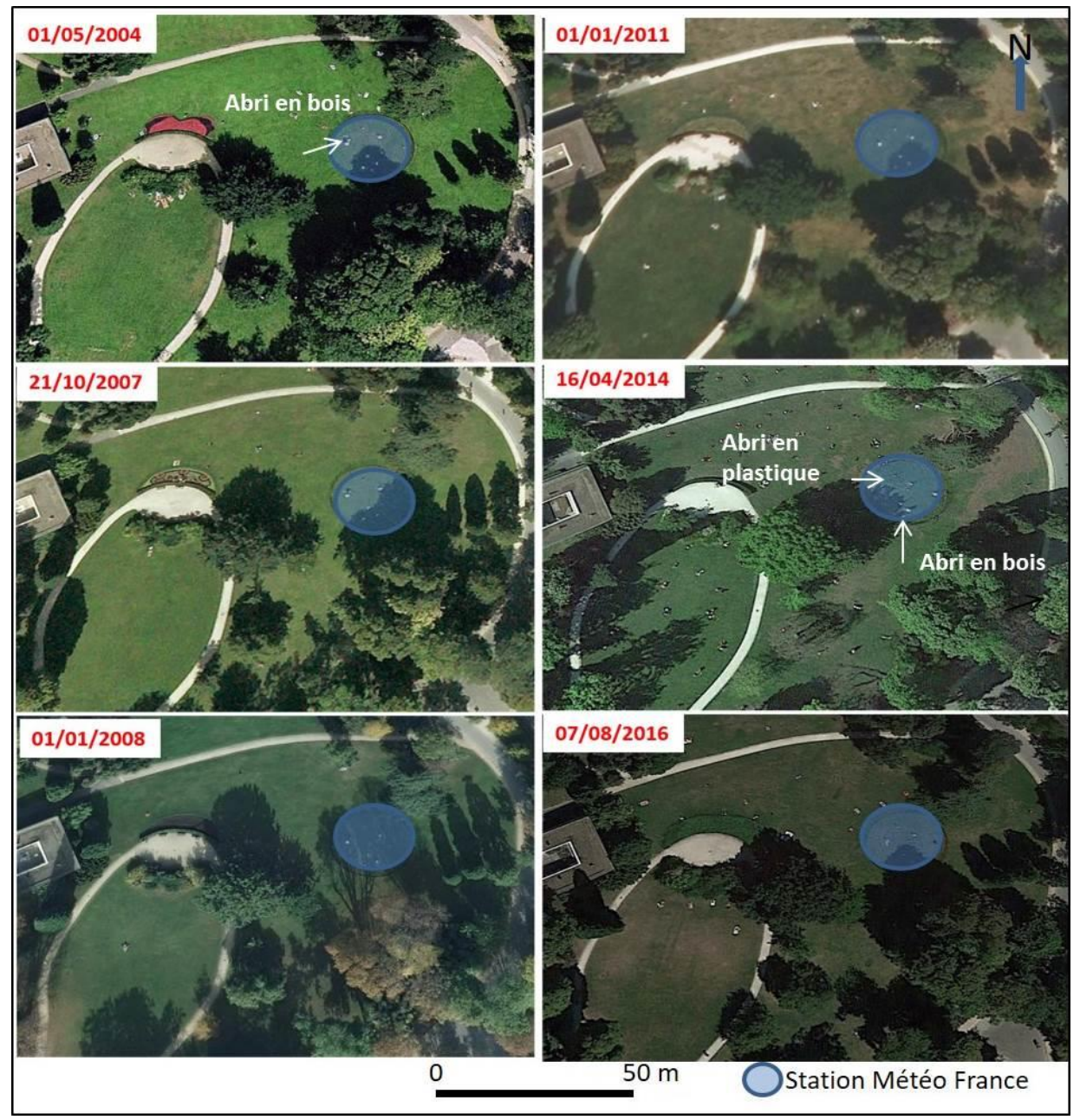

Figure 2. Captures d'écran Google Earth illustrant l'effet de l'ombre sur la station météorologique du parc ParisMontsouris (les capteurs sont visibles sur les photos en annexe 1). Google Earth screenshots illustrating the effect of shadows on the Parc Paris-Montsouris weather station (sensors photos in annexe 1).

Afin d'étudier l'influence de l'ombre sur les températures relevées à Paris-Montsouris, nous avons réalisé une simulation de l'ombre autour de la station, en nous basant sur des données géographiques décrivant l'environnement et à partir de la librairie R shadow (Dorman et al., 2019). Nous avons utilisé R, logiciel de traitement de données, libre et largement répandu, utilisé dans plusieurs études récentes portant sur l'effet de l'ombre sur la température (Massetti et al., 2019 ; Erell et al., 2019 ; Moro et al., 2020).

Outre l'utilisation d'un modèle numérique de terrain donnant l'altitude au sol (RGE ALTI® IGN $1 \mathrm{~m}$ ), nous avons créé plusieurs couches géographiques à partir des observations de terrain et des images satellites de Google Earth, localisant les capteurs météorologiques et délimitant la zone de la station météorologique et l'emprise des arbres renseignée par une hauteur approximative (de 8 à $17 \mathrm{~m}$ ). À partir des emprises extrudées selon leur hauteur, la librairie R shadow permet de calculer la hauteur de l'ombre, son empreinte au sol, la part de ciel visible (sky view factor) ou encore, une estimation du rayonnement solaire tenant compte de l'ombre, tout cela en fonction de la date et de l'heure (avec la librairie R maptools). Plus précisément, nous avons ici simulé l'ombre au niveau du capteur (en prenant donc en compte sa hauteur) puis calculé la part des surfaces ombragées au sol dans un rayon de $5 \mathrm{~m}$ autour, à plusieurs dates et en fonction des heures.

Toujours pour montrer l'effet de l'ombre à la station de Paris-Montsouris, nous avons installé deux stations Davis dans des sites dégagés et exposés au soleil : la première dans le parc même, à moins de $20 \mathrm{~m}$ de celle de Météo France; la 
deuxième dans la Cité Universitaire Internationale de Paris (CUIP) à environ $500 \mathrm{~m}$ (annexe 2). Les mesures ont été réalisées par temps radiatif chaud et clair entre le 25 juin et le 2 juillet 2018. Les stations Davis sont de type Vantage Pro2 mesurant la température et l'humidité de l'air, la pression atmosphérique, le vent et les précipitations. Seuls les deux premiers paramètres sont utilisés ici ; leur résolution et précision sont respectivement de $1^{\prime}$ 'ordre de $0,1^{\circ} \mathrm{C}$ et $0,5^{\circ} \mathrm{C}$ pour les températures, et de 1 et $3 \%$ pour l'humidité relative, d'après le constructeur (davis-meteo.com/PDF/PR57FR.pdf). Les deux stations Davis ont été installées côte à côte, leurs données sont cohérentes et aucun étalonnage additionnel n'a paru nécessaire. Par ailleurs, la station Davis a été installée, pendant 3 jours, à côté de celle de Météo France, comme l'atteste la photo en annexe 2. Un étalonnage de base a été réalisé car les données Davis étaient légèrement plus élevées, sans toutefois dépasser la marge d'erreur du capteur $\left(0,5^{\circ} \mathrm{C}\right)$.

Pour montrer les effets de l'urbanisation et de la végétalisation, nous étudions l'écart thermique enregistré, la nuit, entre Paris-Montsouris et Orly, d'une part. D'autre part, nous réalisons des mesures itinérantes dans l'agglomération parisienne avec une station Testo 400, par temps caniculaire, la nuit du 29 au 30 juin 2019 entre 23 h et 1 h TU. Le capteur utilisé est à la fois précis $\left(0,2^{\circ} \mathrm{C}\right.$ de précision et $0,1^{\circ} \mathrm{C}$ de résolution) et surtout, possède un temps de réponse rapide de l'ordre de quelques secondes, ce qui permet de bien mesurer les variations spatiotemporelles rapides de la température. Le but de ce genre de mesure est de comparer essentiellement les températures du parc Montsouris à celles du centre de Paris ( $1^{\text {er }}$ et $2^{\text {ème }}$ arrondissements). En outre, pendant cette campagne, des mesures supplémentaires dans le parc et ses alentours sont réalisées pour mettre en exergue l'effet combiné de la topographie et de l'occupation du sol sur la température.

\section{Effet de l'ombre sur les températures de l'air}

Il est admis que les températures mesurées par les services météorologiques sont réalisées sous abri pour "éviter son réchauffement par le rayonnement solaire, son refroidissement par les précipitations et l'évaporation de l'eau, son refroidissement nocturne par rayonnement infrarouge » (Météo France, 2011). Parallèlement, les abris doivent être à l'écart de toute ombre portée lorsque la hauteur du soleil est supérieure à $3^{\circ}$ d'après l'Organisation Mondiale de la Météorologie (OMM, 2010). D'après les normes de Météo France et de l'OMM, la distance aux obstacles devrait dépasser 2 fois leur hauteur et idéalement 4 fois. Il est très important que l'abri soit exposé aux divers vents pour favoriser au maximum le renouvellement de l'air (Météo France, 2011).

La station de Paris-Montsouris est entourée par des arbres d'une hauteur variant de 10 à $20 \mathrm{~m}$ (figure 2). Durant les journées radiatives, ces derniers abritent les capteurs des radiations solaires pendant une longue période de la journée, ce qui limite le réchauffement de la surface terrestre, ellemême source de chaleur. De même, les arbres freinent le vent et diminuent le renouvellement de l'air. En consultant la fiche des métadonnées de cette station, on se rend compte que l'effet de l'ombre est signalé suite aux relevés de 2013 et 2018 (« ombres portées présentes si hauteur soleil $\left.>7^{\circ} \gg\right)$. Toutefois, les capteurs sont à la portée de l'ombre bien évidemment avant ces dates, comme en témoignent les figures 1 et 2 . La fiche de métadonnées signale également la présence de sources de chaleur ou d'étendues d'eau à moins de $10 \mathrm{~m}$. Les observations de terrain confirment ce facteur ombre, quelle que soit la saison, à des degrés divers (annexe 1). En effet, les obstacles (arbres) situés au NE, et surtout du SE à l'O des capteurs, les abritent de la radiation solaire le matin et l'aprèsmidi. Les capteurs de températures, pourtant dédoublés depuis quelques années, n'échappent pas à l'effet de l'ombre, quelle que soit la saison (figure 2).

La simulation de l'ombre des arbres sur le capteur, pour une date donnée, permet de visualiser les heures durant lesquelles le capteur est au soleil ou non (deux modalités possibles ; figure 3A) et de quantifier la part de l'ombre au sol dans une zone autour (ici $5 \mathrm{~m}$ ), selon un indice allant de 0 (aucune ombre au sol autour) à $100 \%$ (toute la surface autour est à l'ombre). Par exemple, le 15 juin, le capteur est quelques instants à l'ombre l'après-midi, alors qu'il l'est près de 3 h le 15 août, du fait d'une 
position plus basse du soleil et de la présence d'obstacles (arbres). Lors des après-midis estivaux, la surface autour du capteur (dans un rayon de $5 \mathrm{~m}$ ) connaît une part d'ombre d'au moins $20 \%$ le 15 juin à plus des $2 / 3$ le 15 août, ce qui peut jouer sur les échanges énergétiques et influencer alors la température de l'air mesurée, même sous abri (avec un léger décalage lié à l'inertie thermique).
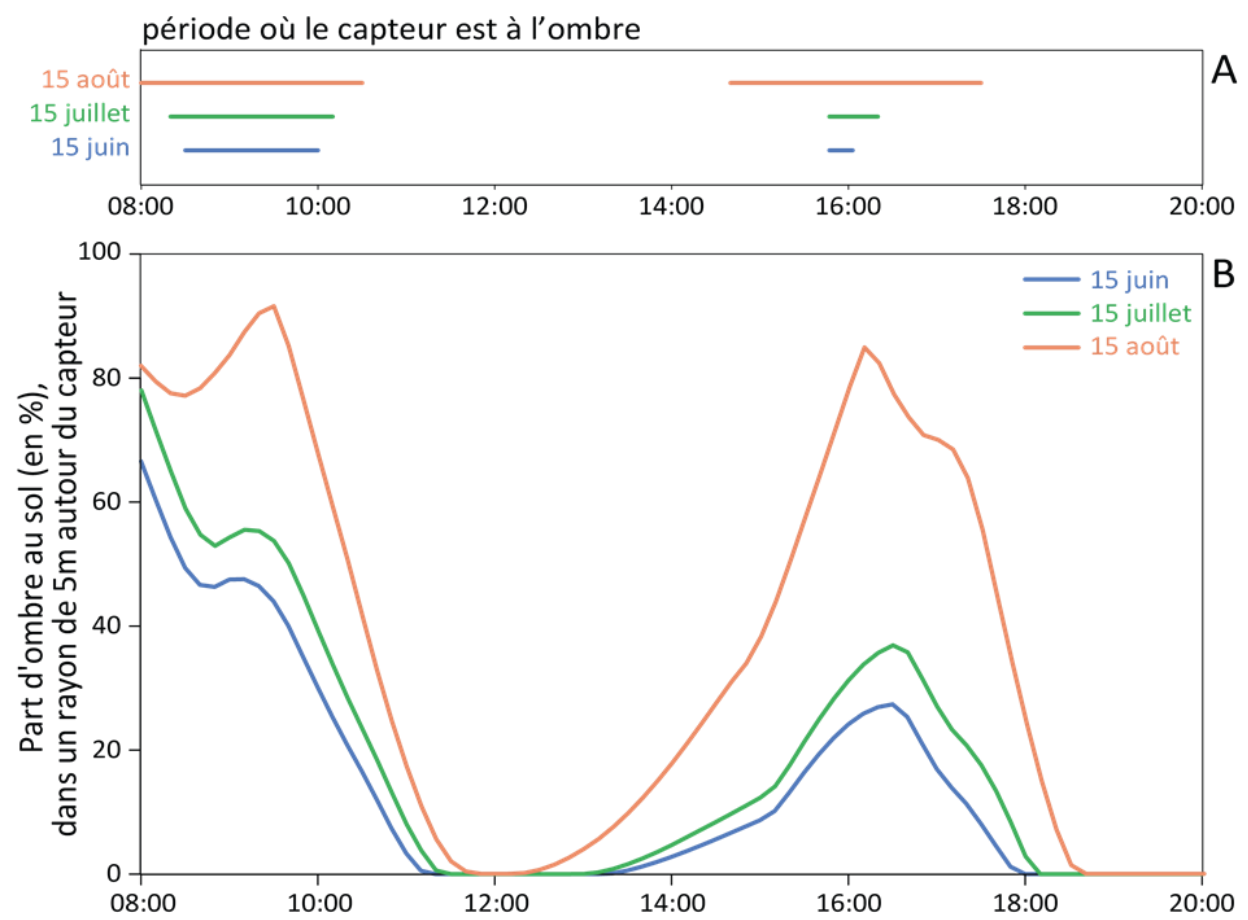

Figure 3. Simulation de l'ombre au niveau du capteur de température pour les trois dates estivales (A) et pourcentage des surfaces ombragées dans un rayon de $5 \mathrm{~m}$ autour du capteur $(\mathrm{B})$. Simulation of the shadow at the temperature sensor for the three summer dates $(A)$ and percentage of shaded areas within $5 \mathrm{~m}$ of the sensor $(B)$.

En été, par temps radiatif (nébulosité $<2$ octas, vent inférieur à $4 \mathrm{~m} / \mathrm{s}$ et pression > à $1013 \mathrm{hPa}$ ), une baisse de la température est observée à Paris Montsouris par rapport aux stations d'Orly et de Vélizy entre 13 h et 18 h TU. En moyenne, entre 1998 et 2017, cet écart atteint son maximum entre $16 \mathrm{~h}$ et $17 \mathrm{~h}$ durant lesquelles il avoisine $-2,5^{\circ} \mathrm{C}$ (figure 4A) et persiste jusqu'à $19 \mathrm{~h}$ (quand la portée des ombres est développée ; figures $4 \mathrm{~A}$ et $5 \mathrm{~A}$ ). Néanmoins, cet écart thermique s'amenuise vers midi quand le soleil est proche de la verticale (figure 4C). L'effet de masque créé par les ombres des arbres situés aux alentours des capteurs de la station Montsouris expliquerait en grande partie les différences de températures entre les deux stations durant l'après-midi.

En été, par temps perturbé, durant les jours caractérisés par une forte nébulosité, un vent relativement fort et une basse pression, le résultat s'inverse: Paris Montsouris devient légèrement plus chaude que les deux autres stations durant l'après-midi (figure 4D). L'effet refroidissant du vent serait moins déterminant dans cette station à cause de la forte rugosité créée par les obstacles : les arbres et les bâtiments situés dans le parc et ses alentours.

En hiver, Paris-Montsouris enregistre des températures plus élevées que les deux autres stations $\left(0,5\right.$ à $\left.1^{\circ} \mathrm{C}\right)$ pour la période $1998-2017$ (figure 4B). Ces variations saisonnières peuvent s'expliquer par la variation de l'intensité de la radiation solaire et la durée de l'ensoleillement (62,5 h en janvier contre 212,5 h en juillet et août, normale 1981-2010 ), la forte occurrence des situations radiatives pendant la saison estivale par rapport à l'hiver (Cantat, 2004) et le ralentissement du vent dans le parc de Montsouris.

\footnotetext{
${ }^{1}$ www.infoclimat.fr/climatologie/normales-records/19812010/paris-montsouris
} 
Figure 4. Cycle quotidien moyen des températures de juillet $(A)$ et janvier (B) enregistrées par les stations de Paris-Montsouris, Orly et Vélizy par temps radiatif et cycle quotidien de l'écart thermique entre les stations de Paris-Montsouris et Orly par temps radiatif $(C)$ et perturbé (D) en été (1998-2017, données METAR, NOAA); d'après Dahech et al. (2018). Mean daily temperatures cycle of July (A) and January (B) recorded by the Paris-Montsouris, Orly and Vélizy stations by nice weather and daily cycle of the temperature difference between the Paris-Montsouris and Orly stations in nice $(C)$ and disturbed $(D)$ weather in summer (1998-2017, NOAA); from Dahech et al. (2018).

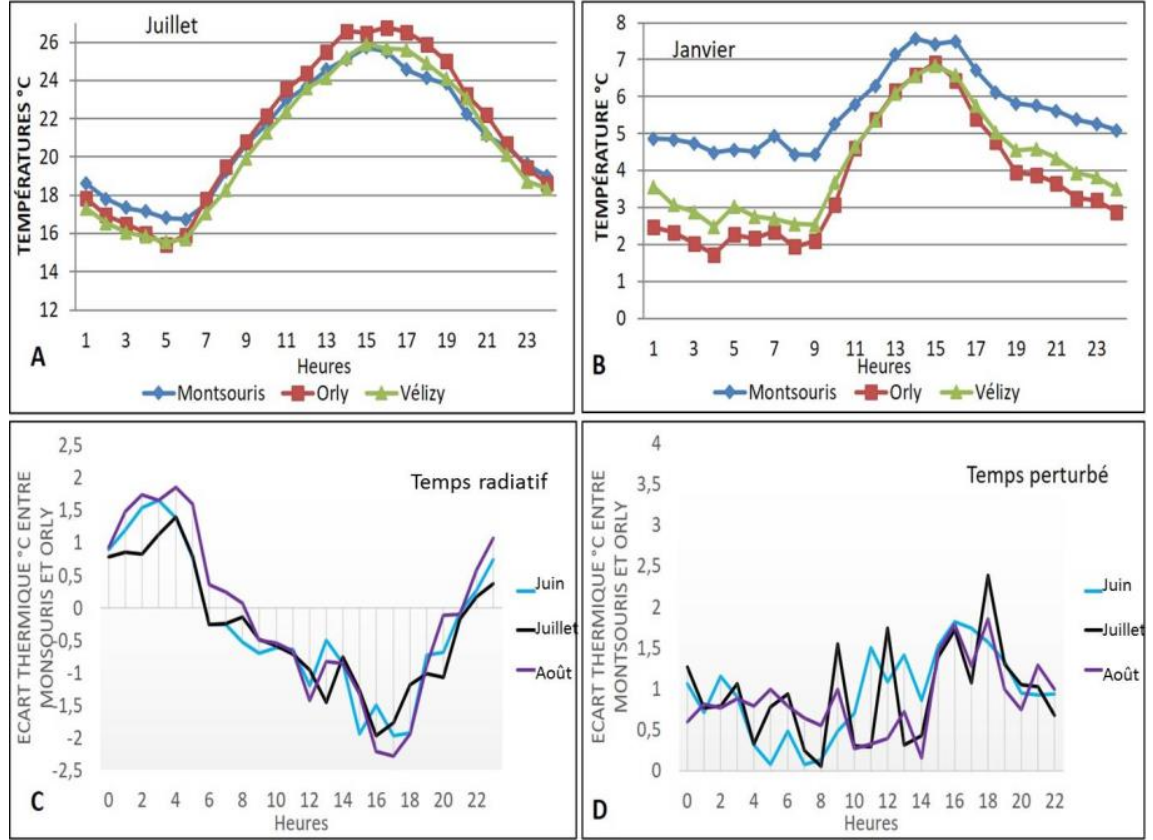

En dehors de l'effet de l'ombre, une baisse des températures peut être expliquée soit par l'effet rafraichissant du vent, soit par un taux d'humidité élevé favorisant la chaleur latente en dépit de la chaleur sensible. Pour vérifier cette hypothèse, nous avons examiné les taux d'humidité, exprimés par la température du point de rosée $(\mathrm{Td})$ et la vitesse des vents dans les stations de Paris-Montsouris et d'Orly. En effet, le vent est freiné dans la première station par la végétation et le bâti entourant le parc : une baisse d'environ $1,5 \mathrm{~m} / \mathrm{s}$ est enregistrée au cours de la journée par rapport à Orly (figure 5A). Cette dernière est, en moyenne, plus humide que

Figure 5. Cycles quotidiens moyens du point de rosée (A) et de la vitesse moyenne du vent (B) par temps radiatif aux stations de Paris-Montsouris et Orly (19982017, données METAR, NOAA). Average daily cycles of dew point $(A)$ and average wind speed $(B)$ in clear weather at the Paris-Montsouris and Orly stations (1998-2017, METAR, NOAA data).
Montsouris l'après-midi : les valeurs de Td sont de $12,2^{\circ} \mathrm{C}$ à Orly en juillet entre 16 et $19 \mathrm{~h}$, contre $11,7^{\circ} \mathrm{C}$ à Montsouris (figure $5 \mathrm{~B}$ ). Ces deux facteurs (humidité et vent plus faibles) favorisent, théoriquement, la hausse de la température à ParisMontsouris et compensent donc vraisemblablement, en partie, l'effet rafraichissant de l'ombre en été. Toutefois, il est très difficile d'évaluer avec précision le poids relatif à chaque facteur ; mais la diminution de la radiation directe semble être parmi les facteurs explicatifs les importants.

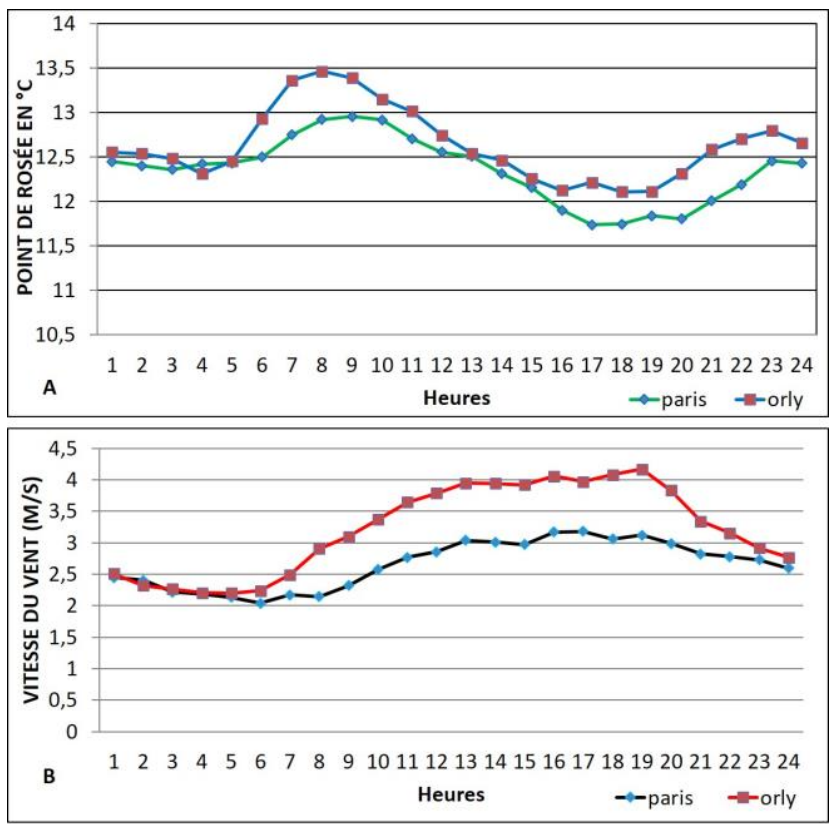


Pour valider ce constat, nous avons installé en juin 2018, une station météorologique fixe de type Davis, à 500 m de la station de Paris-Montsouris, à la Cité Universitaire Internationale de Paris (CUIP), sur une pelouse ensoleillée, durant toute la journée. La comparaison des températures relevées par les deux stations confirme l'effet de l'ombre. Si en phase nocturne, l'écart entre les deux stations s'annule, une baisse des températures d'environ

Figure 6. Températures à Paris-Montsouris et à la Cité Universitaire Internationale de Paris (CUIP) le 30 juin 2018 (en rouge, données issues d'une station Davis). Temperature at Paris-Montsouris and the Cité Universitaire Internationale de Paris (CUIP) on 30/6/2018 (in red data from a Davis station). $1^{\circ} \mathrm{C}$ est affichée par la station conventionnelle très tôt le matin et en fin d'après-midi (figure 6). Les écarts les plus marqués sont enregistrés quand les rayonnements solaires sont obliques, lorsque les ombres portées sont étendues et couvrent les capteurs (figure 3). Le maximum de l'écart avoisine $1,8^{\circ} \mathrm{C}$ vers $19 \mathrm{~h}$ TU. À cette heure, la station d'Orly enregistre pareillement un écart positif d'environ $1^{\circ} \mathrm{C}$ par rapport à Montsouris.

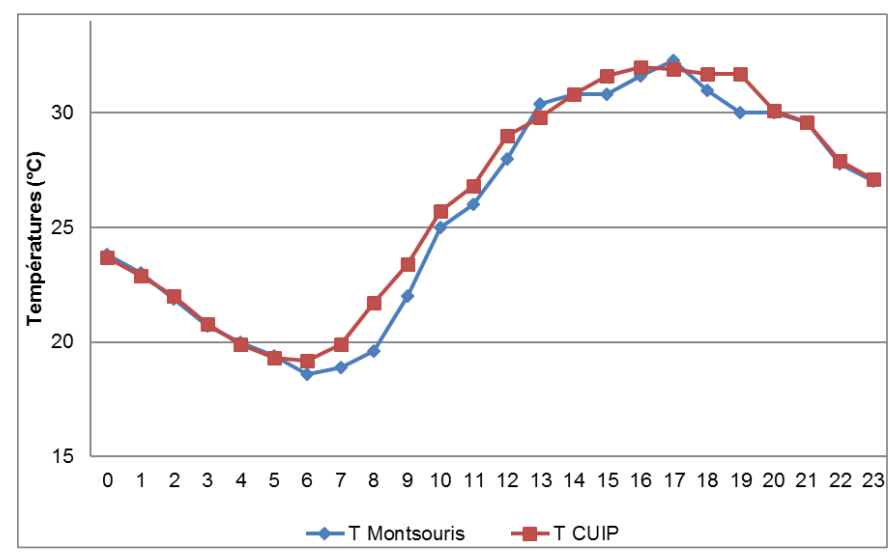

rayon de $1 \mathrm{~km}$ de l'abri météorologique, sur une colline, pas vraisemblablement représentative de l'ambiance urbaine. Le but de cette étude étant de monter la représentativité des données (températures) de cette dernière station, la comparer avec Orly pourrait être justifiable. Même si les données de ces deux stations ne permettent pas d'étudier l'intensité maximale de l'ICU en région parisienne, le calcul du gradient thermique entre les deux stations, exclusivement par temps radiatif, montre quand même l'effet de la ville en phase nocturne. La nuit, les températures sont plus élevées à Montsouris. La ville est plus chaude car les surfaces artificielles stockent une grande quantité de radiation solaire incidente qui sera restituée la nuit ; elle se caractérise par la faiblesse de la chaleur latente, malgré l'effet du parc, contrairement à la campagne (Oke, 1982). L'intensité de l'ICU, connu comme un phénomène nocturne de temps radiatif, varie temporellement d'une saison à autre (figure 7) : il dure évidemment plus longtemps en hiver. L'écart entre Paris-Montsouris et Orly ne dépasse pas $5^{\circ} \mathrm{C}$, en moyenne, par temps radiatif. Il est atteint en hiver, ce qui pourrait être justifié par les activités anthropiques et le fort refroidissement par temps clair en périphérie (figure 7). 
Figure 7. Variation mensuelle du cycle journalier $(24 \mathrm{~h})$ des écarts thermiques entre la station de Paris-Montsouris et celle d'Orly par temps radiatif (1998-2017, données METAR, NOAA). Monthly variation of the daily thermal differences cycle (24h) between the Paris-Montsouris station and the Orly station by nice weather (1998-2017, METAR data, NOAA).

Afin d'évaluer la représentativité spatiale du site de la station Paris-Montsouris, nous avons réalisé une campagne de mesure itinérante, la nuit du 29 au 30 juin 2019 entre 23 h et $1 \mathrm{~h}$ TU, montrant la variation spatiale des températures dans le parc et ses alentours, et mesurant le gradient entre le centre de Paris et sa campagne environnante au sud de l'agglomération. Le 29 juin, le ciel était clair, la température maximale a atteint $36,5^{\circ} \mathrm{C}$, soit un record jamais atteint dans cette station en juin. Les températures variaient de 26,4 à $26^{\circ} \mathrm{C}$, avec un vent faible, inférieur à $3 \mathrm{~m} / \mathrm{s}$, et un indice de confort humidex (température ressentie) de $31^{\circ} \mathrm{C}$ relevés entre 23 h et $1 \mathrm{~h}$ TU par la station de Météo France. Des rectifications légères $\left(<0,5^{\circ} \mathrm{C}\right)$ sont apportées aux valeurs enregistrées pendant la campagne de mesures itinérantes pour annuler l'effet de la variation temporelle des températures de l'air.

D'après les mesures mobiles, les températures sont hétérogènes dans le parc Montsouris. Elles varient de 25 à $27,5^{\circ} \mathrm{C}$ entre $23 \mathrm{~h} 30$ et $0 \mathrm{~h}$ TU (figure 8A). La partie située au nord-ouest du parc, relativement surélevée du point de vue topographique et située en aval de constructions denses, est relativement plus chaude. Cependant, la partie vallonnée, située au nord-est, à proximité du lac artificiel, est la plus fraîche. Une légère brise fraîche dont la vitesse est inférieure à $1,5 \mathrm{~m} / \mathrm{s}$ diverge du parc vers les quartiers denses situés du $\mathrm{NE}$ au S du parc. Cependant, nous avons remarqué l'advection d'un air relativement chaud, au niveau de certains endroits proches de la bordure nordouest du parc, canalisé par les canyons urbains (rue square du parc) et favorisé par la pente. Près des capteurs de la station de Météo France, la température est d'environ $26,5^{\circ} \mathrm{C}$, soit une valeur parmi les plus élevées dans le parc à cause de la

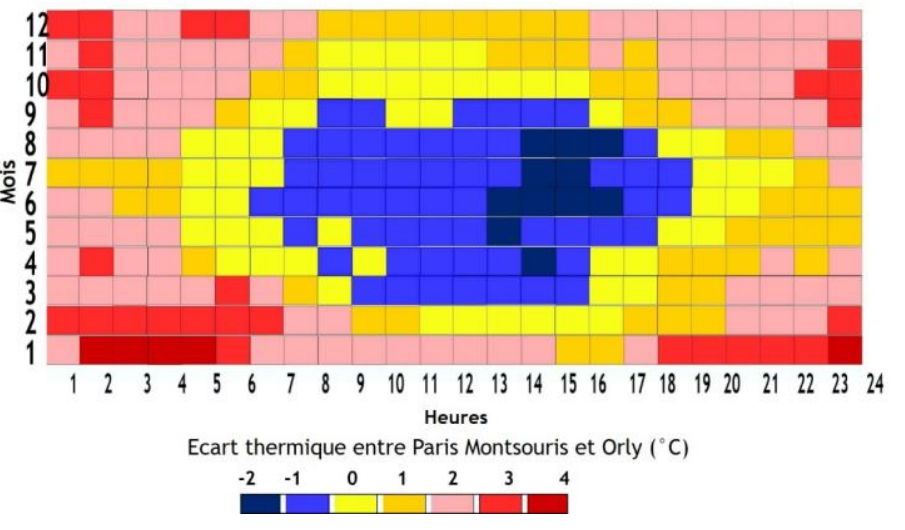

topographie du point de mesure et la faible distance aux bâtiments. En effet, la station est située à $75 \mathrm{~m}$ d'altitude, sachant que dans le parc, la topographie varie de 82 à $53 \mathrm{~m}$. De plus, la station est située à $60 \mathrm{~m}$ d'un bâtiment et à $100 \mathrm{~m}$ du boulevard des Maréchaux. Dans les quartiers avoisinant le parc, les températures oscillent entre 28 et $29^{\circ} \mathrm{C}$ (14 et $13^{\text {ème }}$ arrondissements) dans un rayon de $1 \mathrm{~km} \mathrm{du}$ parc. La même nuit, pendant cette campagne, les températures ont atteint $32,5^{\circ} \mathrm{C}\left(1^{\mathrm{er}}\right.$ arrondissement de Paris) et $21,5^{\circ} \mathrm{C}$ en plein champ de maïs à environ $15 \mathrm{~km}$ au sud de Paris, à Epinay sur Orge (figure 8B). Dans cette commune, les vallées de l'Orge et de l'Yvette enregistrent une baisse moyenne de $2^{\circ} \mathrm{C}$ par rapport au plateau sous l'effet combiné de l'occupation du sol et la topographie. En effet, l'air froid s'accumule au fond des vallées, souvent végétalisées, alors que dans le centre de la commune, en haut du plateau, les surfaces bâties modifient le bilan d'énergie près du sol. Elles stockent plus de chaleur durant la journée : les surfaces artificielles consomment plus de chaleur sensible au détriment de la chaleur latente et la restituent la nuit sous forme de rayonnement tellurique (Oke, 1987), d'où l'augmentation des températures la nuit.

\section{Discussion}

L'effet de l'ombre à Montsouris peut sousestimer les températures quotidiennes maximales, souvent utilisées pour étudier la tendance des températures à l'échelle pluriannuelle dans cette station centenaire. Afin d'éviter l'effet de l'ombre dans le parc Montsouris, nous suggérons de dédoubler le capteur de température. Le nouveau site proposé, à $25 \mathrm{~m}$ au NO du présent emplacement, est non influencé par l'ombre portée (annexe 3). 
Figure 8. Répartition spatiale des températures de l'air dans le parc Montsouris (A) et le long d'un transect Châtelet/Epinay-sur-Orge (B) par temps caniculaire (mesures itinérantes réalisées la nuit du 29-30 juin 2019 entre 23 h et $1 \mathrm{~h}$ TU du matin, méthode d'interpolation Spline) ; cartographie : R. Bouaziz et S. Dahech. Spatial distribution of air temperatures in Montsouris Park (A) and along a Chatelet/Epinay-sur-Orge transect $(B)$ in very hot weather (car survey carried out on 29-30 June 2019 between $11 \mathrm{pm}$ and 01 am, by spline interpolation method).

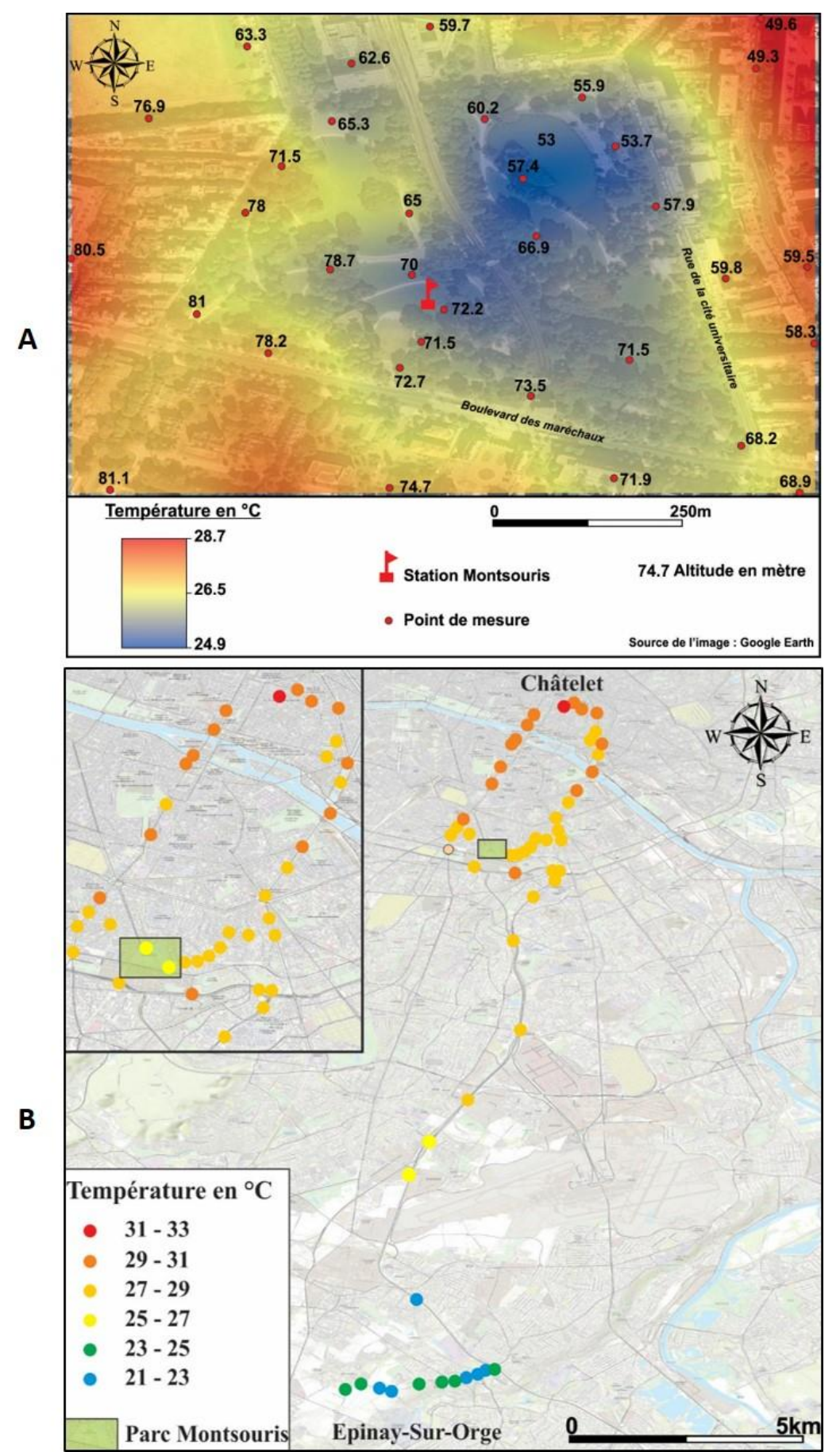

Le site choisi pour implanter la station météorologique automatique Davis à la CUIP présente l'avantage d'être proche de la station Montsouris, à l'écart de l'ombre portée, installée sur une pelouse entretenue qui s'étend sur environ $100 \mathrm{~m}^{2}$. Cependant, la végétation est entrecoupée par un chemin caillouteux situé à environ $1 \mathrm{~m}$ à l'est et au nord de la station, qui pourrait, le matin, influencer sensiblement la température dans l'environnement proche. Ce fait peut expliquer, en partie, la légère hausse des températures dans la station du CUIP entre 7 et $10 \mathrm{~h}$, mais ne peut empêcher l'impact de l'ombre à Montsouris le matin. En effet, durant l'épisode anticyclonique du $1^{\text {er }}$ au 21 septembre 2020 , une baisse de $0,9^{\circ} \mathrm{C}$, en moyenne, est enregistrée par rapport à Orly entre 8 et $12 \mathrm{~h}$. Cet écart devient positif $\left(1,5^{\circ} \mathrm{C}\right.$ en moyenne) la nuit. Nous suggérons la grande pelouse comme site idéal pour implanter une station météorologique automatique dans la CUIP (annexe 3). Malheureusement, ce site a été inaccessible durant la campagne de juin-juillet 2018. 
Les mesures itinérantes ont permis de mesurer, entre autres, la température dans la partie la plus chaude de l'agglomération. Le 30 juin 2018, vers $23 \mathrm{~h} 30 \mathrm{TU}$, nous avons relevé une température de $32,2^{\circ} \mathrm{C}$ et une humidité relative de $49 \%$ sur le Boulevard de Sébastopol, ce qui ramène la température ressentie, d'après l'indice de confort humidex, à $40^{\circ} \mathrm{C}$. Il est évident que la station de Montsouris ne permet pas de nous renseigner sur l'ambiance thermique dans la partie dense de l'agglomération parisienne et que des données à échelle fine (issues de la mesure ou de la modélisation) sont requises pour adopter les solutions adéquates en situation de canicule.

Pour pallier le caractère ponctuel de la campagne de mesures itinérantes décrite dans ce travail, une deuxième a été réalisée le 12 octobre 2019 par temps radiatif avec un vent faible du secteur méridional. Elle confirme les résultats obtenus. Toutefois, par rapport au 30 juin 2019, journée caniculaire, les écarts thermiques sont plus modérés : l'intensité de l'ICU n'a pas dépassé $8^{\circ} \mathrm{C}$; un écart de $3^{\circ} \mathrm{C}$ entre le centre de Paris et le parc Montsouris et environ $1{ }^{\circ} \mathrm{C}$ de différence à $1^{\prime}$ intérieur du parc sont mesurés.

\section{Conclusion}

Cette étude confirme que les stations dites « de référence » sont souvent seulement et uniquement l'image climatologique de leur environnement immédiat et qu'une généralisation sur l'ensemble d'une région est impropre. À Paris-Montsouris, les arbres entourant l'abri météorologique créent de l'ombre et influencent donc les températures enregistrées. Dans cette station, une baisse de la température par rapport aux stations d'Orly et de Vélizy, situées en milieu dégagé, a été observée entre $14 \mathrm{~h}$ et $19 \mathrm{~h}$, notamment en été par temps radiatif. Dans le parc de Montsouris, les températures sont hétérogènes. Durant une nuit estivale caniculaire, les mesures par capteurs portables ont révélé une variation d'environ $2,5^{\circ} \mathrm{C}$ dans le parc en fonction de la topographie et de l'occupation du sol. Durant la même nuit, vers minuit, le centre de Paris enregistre $5^{\circ} \mathrm{C}$ de plus que la station de Paris-Montsouris, et $12^{\circ} \mathrm{C}$ de plus que la campagne, au sud, en Essonne.
Cette étude prouve que la température mesurée dans la station de Paris-Montsouris ne représente que le parc urbain dans lequel elle est située, voire même une partie du parc. Il s'agit de la température de l'air urbain à Paris, influencée par l'effet de site (parc urbain sur une colline). Pour cette raison, le recours à la chronique de cette station pour des études dynamiques exige beaucoup de prétraitement avant d'analyser l'évolution « naturelle » du climat. Pour mieux cerner les effets du site, il serait intéressant de comparer la température à cette station à d'autres implantées en milieu rural le plus proche et en milieu urbain non végétalisé, sur une période assez longue, de l'ordre d'au moins une année.

Remerciements : Les auteurs adressent leurs vifs remerciements aux collègues qui ont participé aux campagnes de mesures: Hamdi Euchi, Marwen Ghribi, Mohamed Chebli, Riadh Bouaziz et Mohamed Feki.

\section{Références}

Beltrando G., Dahech S. et Madelin M., 2008. L'intérêt de l'étude des brises thermiques : exemples des brises littoraux et orographiques. Bulletin de la Société Géographique de Liège, 51, 49-61.

Cantat O., 2004. L'îlot de chaleur urbain parisien selon les 'types de temps'. Norois, 191, 75-102.

Carrega P., 1994. Topoclimatologie et Habitat. Thèse, Revue de géographie du laboratoire d'analyse spatiale Raoul Blanchard n 35 et 36, Université de Nice, France, 408 p.

Charfi S., 2012. Le comportement spatio-temporel de la température dans l'agglomération de Tunis. Thèse de doctorat, Université de Tunis et Université de Nice-Sophia Antipolis (en cotutelle), $308 \mathrm{p}$.

Charfi S., Krout H. et Dahech S., 2014. Vegetation in the urban area of Tunis: a study of impacts on temperature and light. Climatologie, 11, 72-78.

Dahech S., 2013. Le réchauffement contemporain en Tunisie (1960-2012): rôle de la circulation atmosphérique et de l'urbanisation. Journées de la Commission Climat et Société du Comité National Français de Géographie, 14-16 mars, Epernay, 68-88.

Dahech S., Charfi S. et Madelin M., 2018. Impact de l'environnement immédiat des stations météorologiques sur la mesure de la température. Acte du XXXI ${ }^{\text {me }}$ de colloque de l'Association Internationale de Climatologie, Nice, 14-20.

Dorman M., Erell E., Vulkan A. et Kloog I., 2019. Shadow: R Package for Geometric Shadow Calculations in an Urban Environment. The R Journal, 11.1, 287-309. 
Douguedroit A. et Bridier S., 2007. Réchauffement climatique et évolution de l'environnement des stations en région méditerranéenne française (1951-2000). Actes du XXème Colloque de l'Association Internationale de Climatologie, Carthage, Tunisie, 3-8 septembre 2007, 204- 209.

Erell E., Dorman M., Alexandroff V and Kloog I., 2019. Implementing an open-source tool for modelling solar PV potential in dense urban areas. J. Phys.: Conf. Ser. 1343012092. 7p. Doi:10.1088/1742-6596/1343/1/012092.

Escourou G., 1991. Le climat de la ville. Paris, Nathan, 192 p. Girault A., Kounkou-Arnaud R., 2018. Paris face au changement climatique: Actualités, les prévisions saisonnières juin-juillet-août. Les petits déjeuners de presse Météo France et l'Agence Parisienne du Climat, 53 p. apcparis.com/system/files/file_fields/2018/06/12/petit-dejeunerpresse-meteo-france-apc-cc-paris-presentation.pdf.

Golden J. S., 2004. The built environment induced Urban Heat Island effect in rapidly urbanizing arid regions - A sustainable urban engineering complexity. Environmental Sciences, 1(4), 321-349.

Gubler S., Hunziker S., Begert M., Croci-Maspoli M., Konzelmann T., Brönnimann S., Schwierz C., Oria C. and Rosas G., 2017. The influence of station density on climate data homogenization. Int. J. Climatol., 37, 4670-4683.

Huang Y. J., Akbari H., Taha H. and Rosenfeld A. H., 1987. The potential of vegetation in reducing summer cooling loads in residential buildings. American meteorological society, 26, 1103-1116.

Lemonsu A., Kounkou-Arnaud R., Desplat J. Salagnac J.-L. et Masson V., 2013. Evolution of the Parisian urban climate under a global changing climate. Climatic Change, 116, 679692.

Li X., Zhou Y, Yu S., Jia G., Li H., Li W., 2016. Urban heat island impacts on building energy consumption: A review of approaches and findings. Energy, 174, 407-419.

Lockhart T., 2003. Challenges of Measurements. In Handbook of Weather, Climate and Water, chapter 33, Wiley \& Sons. 695-710.

Lowe S. A., 2016. An energy and mortality impact assessment of the urban heat island in the US. Environmental Impact Assessment Review, 56, 139-144.
Martin N., 2008. La pollution par l'ozone et la climatologie dans un espace méditerranéen: Les Alpes-Maritimes. Thèse de doctorat, Université de Nice-Sophia Antipolis, 281 p.

Massetti L., Petralli M., Napoli M. et al., 2019. Effects of deciduous shade trees on surface temperature and pedestrian thermal stress during summer and autumn. Int. J. Biometeorol., 63, 467-479. https://doi.org/10.1007/s00484019-01678-1.

Masson V., Lion Y., Peter A., Pigeon G., Buyck J., Brun E., 2013. Grand Paris: regional landscape change to adapt city to climate warming. Climate Change, 117 (4), 769-782. http://dx.doi.org/10.1007/s10584-012-0579-1.

Météo France, 2011. Conseils sur l'installation de capteurs météorologiques chez des passionnés. Note technique rédigée par Gaëtan Leches, $10 \mathrm{p}$.

Moro J., Krüger El. et Camboim S., 2020. Shading analysis of urban squares using open-source software and free satellite imagery. Appl. Geomat. https://doi.org/10.1007/s12518-02000313-8.

Oke T. R., 1982. The Energetic Basis of the Urban Heat Island. Quarterly Journal of the Royal Meteorological Society, 108, 1-24.

Oliveros J. M., Vallar E. A., Galvez M. C. D., 2019. Investigating the effect of urbanization on weather using the weather research and forecasting (WRF) model: A case of Metro Manila, Philippines. Environments, 6, 10, 17 p.

Organisation Mondiale de la Météorologie (OMM), 2010. Guide des instruments et des méthodes d'observation météorologiques. $\mathrm{N}^{\circ} 8,742 \mathrm{p}$.

Satamouris M., 2014. Cooling the cities - A review of reflective and green roof mitigation technologies to fight heat island and improve comfort in urban environments. Solar Energy, 103, 682-703.

Taha H., 1997. Urban climates and heat islands - Albedo, evapotranspiration and anthropogenic heat. Energy and Buildings, 25, 99-103.

Weng Q., Lu, D. and Schubring J., 2004. Estimation of Land Surface Temperature - Vegetation abundance relationship for Urban Heat Islands. Remote Sensing of Environment, 89, 467483.

Citation de l'article : Dahech et al., 2020. Représentativité des températures mesurées dans la station météorologique Paris-Montsouris. Climatologie, 17, 5 . 


\section{Annexes}

Annexe 1 : Environnement immédiat de la station Paris Montsouris (clichés : S. Dahech). Immediate environment of Paris Montsouris weather station (photos: S. Dahech).
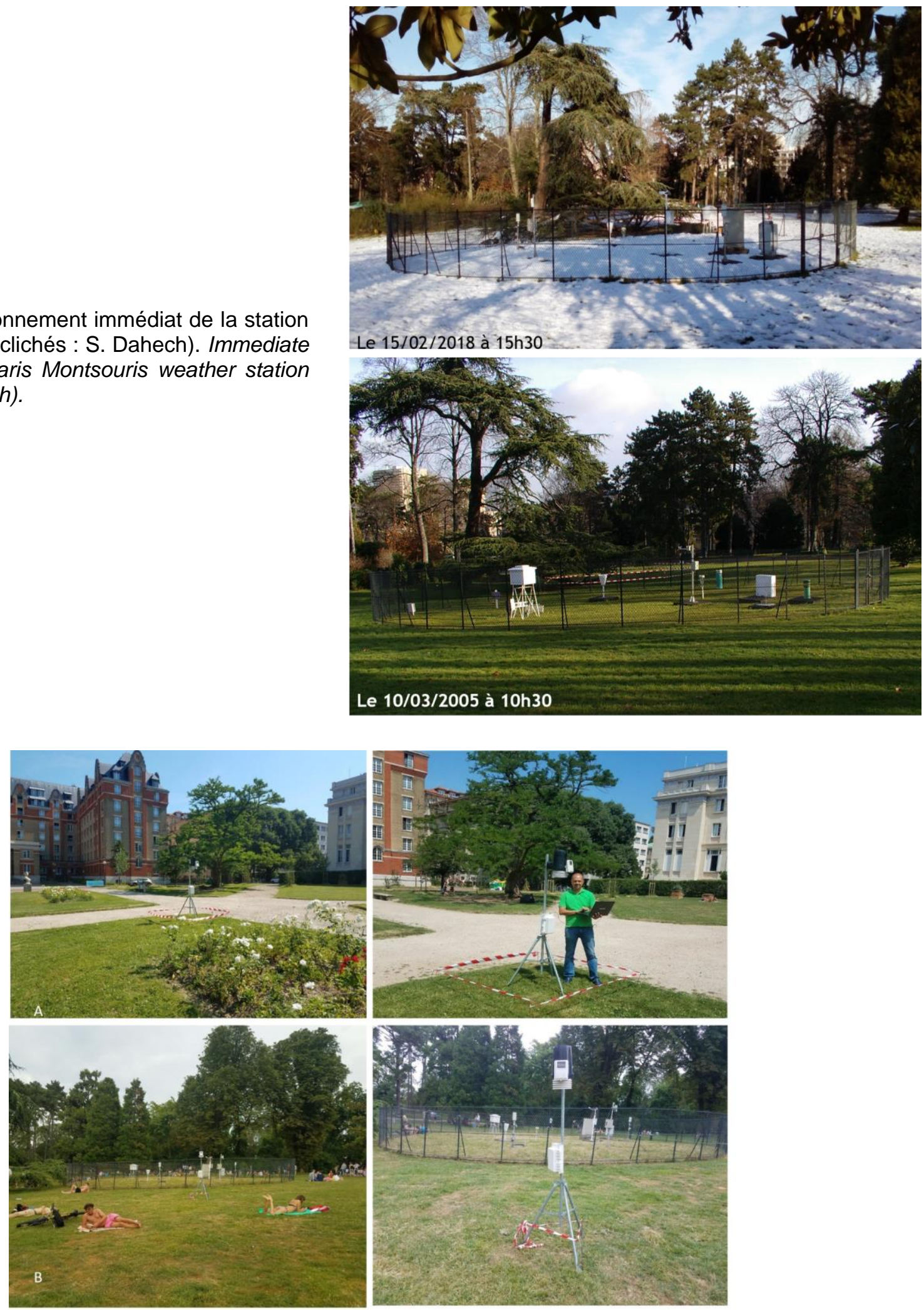

Annexe 2. Photos illustrant le milieu environnant de la station Davis installée dans la cité universitaire de Paris $(A)$ et le parc Montsouris (B) (Clichés Dahech et Charfi, juin 2018). Photos illustrating the environment surrounding the Davis station installed in the CUIP $(A)$ and Montsouris Park (B) (photos: Dahech and Charfi, June 2018). 


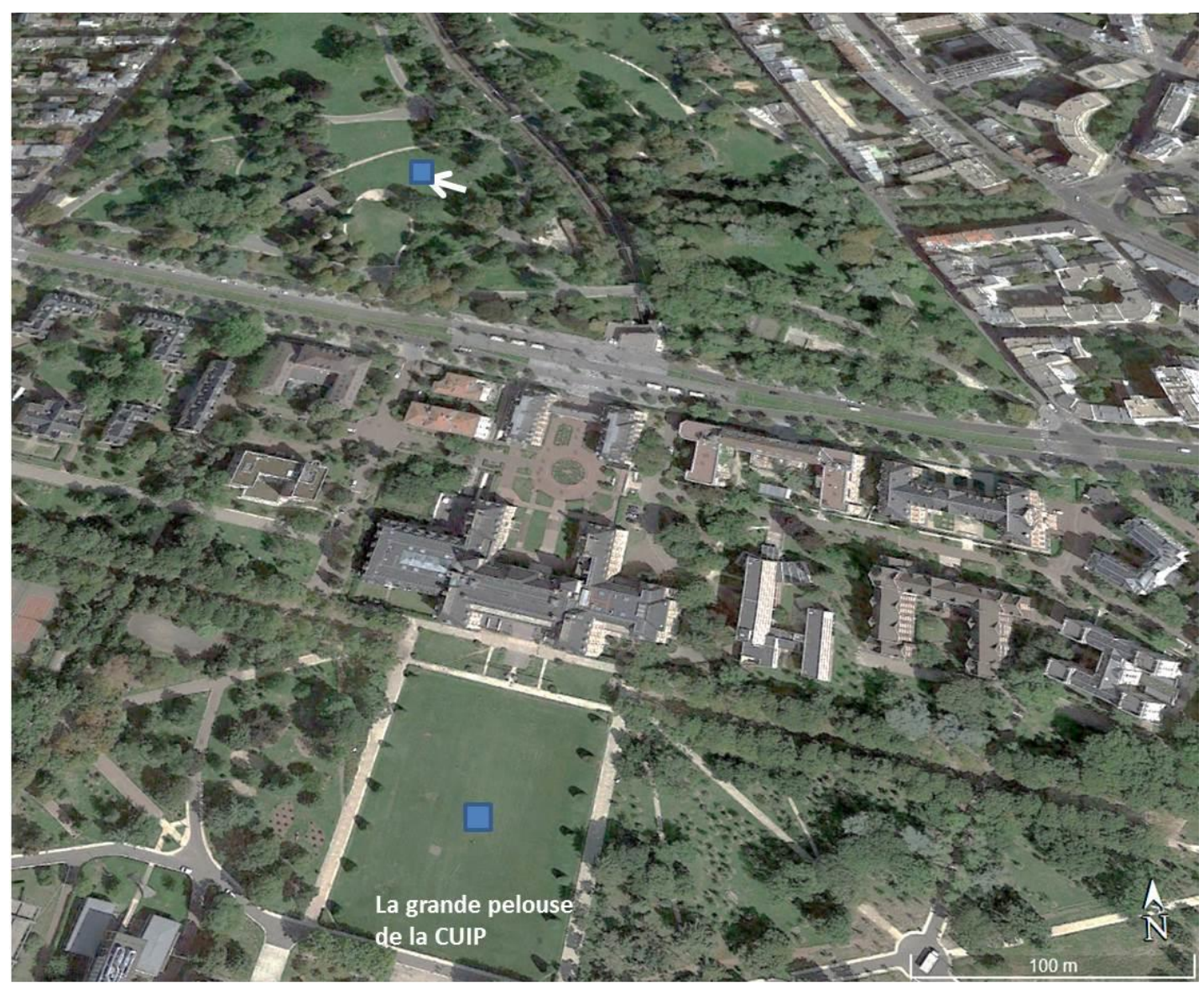

Sites proposés pour implanter une station météorologique

Annexe 3. Nouveaux sites proposés pour implanter deux stations météorologiques dans le parc Montsouris et à la CUIP (source : Google Earth). Proposed new sites of two weather stations in Montsouris Park and at the CUIP (source: Google Earth). 